\title{
Oxaliplatin-Induced Thrombotic Microangiopathy in a Patient with Stage IV Gallbladder Carcinoma: Primary Association or Multiple Hits?
}

\author{
María Cynthia Fuentes-Lacouture Edgar Camilo Barrera-Garavito \\ Internal Medicine Department, Fundación Cardioinfantil, Bogotá, Colombia
}

\section{Keywords}

Thrombotic microangiopathies · Oxaliplatin · Neoplasm · Chemotherapy

\begin{abstract}
Thrombotic microangiopathies (TMA) include a variety of vascular disorders characterized by the presence of microthrombi, coagulopathy by platelet activation and consumption, and systemic damage. The most frequent secondary causes are infections and some medications. However, the presence of chemotherapeutic agents is not so common, and the induction of TMA by oxaliplatin is poorly understood, with few published case reports. We present the case of a patient with a history of gallbladder carcinoma, in whom findings compatible with TMA were documented, and with a temporal and sole relation to oxaliplatin.
\end{abstract}

\section{Introduction}

Thrombotic microangiopathies (TMA) are disorders characterized by intravascular destruction of red blood cells, thrombocytopenia due to activation and consumption, and formation of microthrombi in the systemic vasculature, causing ischemic organ damage [1]. It can be classified as primary or secondary, with thrombotic thrombocytopenic purpura and hemolytic uremic syndrome being the most representative of the former. Secondary causes are often related to acute infections, including HIV infection, and drug-induced ones. Among the latter, some chemotherapeutic agents have been characterized by inducing this clinical 
picture, such as gemcitabine [2]. However, there are few reports about the relationship with oxaliplatin, and apparently none reported at the moment with capecitabine. We present the case of a patient treated with the capecitabine and oxaliplatin (CapeOX) for metastatic gallbladder carcinoma, in whom criteria for chemotherapy-induced TMA were documented.

\section{Case Description}

A 64-year-old female patient consulted the emergency department due to a 5-day history of asthenia and severe abdominal pain associated with 1 episode of dark stools. She had a history of hypertension, type 2 diabetes mellitus, and stage IV T4N1M1 gallbladder carcinoma, with metastatic liver disease, for which she had received chemotherapy initially with gemcitabine and cisplatin; however, due to disease progression she was on CapeOx therapy (capecitabine and oxaliplatin), the last dose 20 days ago. On physical examination, she was pale and jaundiced. The abdominal examination revealed a palpable, $10-\mathrm{cm}$ diameter stone mass located in the right hypochondrium with extension to the epigastrium.

The initial laboratory results showed metabolic acidemia with severe hyperlactatemia; complete blood count revealed leukocytes of $41,600 \mathrm{cell} / \mathrm{mm}^{3}$, with neutrophilia, macrocytic anemia, and severe thrombocytopenia with counts below $30,000 \mathrm{cell} / \mathrm{mm}^{3}$. Kidney function was preserved. Sepsis of abdominal origin was suspected as the first possibility, and antibiotics therapy was started at ICU. An abdominal CT scan revealed a solid hypodense mass in liver segment VI, in close contact and loss of cleavage plane with gallbladder, measuring 100 $\times 86 \mathrm{~mm}$. Because of dark stools and anemia, endoscopic studies of gastrointestinal tract were requested, which were negative for active or recent bleeding. Despite antibiotic management, the patient status worsened, and anemia and thrombocytopenia persisted, requiring continuous transfusion support. Peripheral blood smear was positive for schistocytes. Lactate dehydrogenase level was $978 \mathrm{U} / \mathrm{L}$, indirect bilirubin $1.5 \mathrm{mg} / \mathrm{dL}$, and reticulocyte was elevated, and haptoglobin level was less than $8 \mathrm{mg} / \mathrm{dL}$, confirming hemolytic anemia. The patient was additionally suffering from neurological disorders, caused by mutism and disorientation.

Additional workup showed coagulation abnormalities with consumed fibrinogen. With a plasmic score of 5 points, the diagnosis of TMA was considered taking into account the International Working Group criteria. TMA was suspected to be in association with oxaliplatin and active neoplastic disease. Management with plasmapheresis was proposed; however, in the face of advanced neoplastic disease and secondary complications, it was deferred. The patient died 17 days after admission.

\section{Discussion}

TMA are diseases of the vascular system that cause multi-organic involvement. They are characterized by the presence of microangiopathic anemia, thrombocytopenia, and organ damage [1]. Despite a clear pathophysiology with compromised vasculature, the causes and triggers differ between subgroups and classification, and are thus divided into primary and secondary or acquired. Among the primaries, we are familiar with thrombocytopenic thrombotic purpura, with an incidence of 3 in 1,000,000 inhabitants [3], and hemolytic uremic syndrome.

However, secondary TMA represents the vast majority of cases, reaching $94 \%$ of cases in a retrospective study carried out by Bayer et al. [4]. In this study, it was found that pregnancy, infections, medications, and malignancy were the principal triggers.

\section{Karger'}




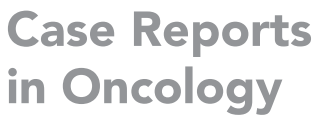

\begin{tabular}{|c|c|}
\hline \multicolumn{2}{|c|}{ Case Rep Oncol 2020;13:1191-1195 } \\
\hline DOI: 10.1159/000510307 & $\begin{array}{l}\text { (c) } 2020 \text { The Author(s). Published by S. Karger AG, Basel } \\
\text { www.karger.com/cro }\end{array}$ \\
\hline
\end{tabular}

Table 1. Clinical characteristics and outcomes of patients with TMA secondary to oxaliplatin in published case reports

\begin{tabular}{llll}
\hline & Baretta Z et al., 2013 & Niu J and Mims MP, 2012 & Dahabreh I et al., 2006 \\
\hline Sex & Male & Female & Male \\
Age & 73 & 68 & 52 \\
Neoplasia & IIIB colon adenocarcinoma & Rectal adenocarcinoma & Sigmoid adenocarcinoma \\
Timing & Fourth cycle & Second cycle & Fourth cycle \\
Treatment & Steroids, frozen plasma infusion & Steroids, plasma exchange & Steroids, frozen plasma infusion \\
Outcome & Death & Complete remission & Complete remission \\
\hline
\end{tabular}

Among drugs associated with TMA, there is a strong association with gemcitabine and calcineurin inhibitors $[4,5]$, frequently used as chemotherapy and immunosuppressants in solid-organ transplant recipients, respectively. In the case of malignancies, the majority of cases represent adenocarcinomas, with another large part being secondary to hematologic diseases. The incidence of TMA reported among these entities ranges between 3 and $12 \%$ [6].

Regarding gemcitabine-induced TMA, a variable time course between the administration of the drug and the development of symptoms has been described, with a mean time at onset of 7.4 months after the administration of gemcitabine. Nevertheless, some authors have tried to identify risk factors for its development, with a median cumulative dose of $20,000 \mathrm{mg} / \mathrm{m}^{2}$ or 21.9 doses being one of the main factors associated with its development [7].

In this case, our patient had a previous diagnosis of gallbladder carcinoma, for which she had previously received gemcitabine therapy, and was currently under management with oxaliplatin because of disease progression. Despite not having ADAMTS13 measurement, with a plasmic score of 5 there was an intermediate risk for the development of TMA, and in the appropriate clinical context and without any other explanation for the patient's manifestations, this diagnosis was considered as a first possibility.

There are some case reports in the literature in which the administration of oxaliplatinbased chemotherapy has been associated with the appearance of TMA [8], in which pathophysiology is still unknown. However, in the three similar reports found in the literature (Table 1), standard treatment with steroids and free frozen plasma or plasma exchange was administered, with 2 of the 3 cases achieving complete remission.

We found no reports in which patients had previous exposure to gemcitabine, with subsequent administration of oxaliplatin, as in our case. This leads us to question the possibility that previous exposure to this drug has favored endothelial changes in our patient that could have facilitated the development of this fatal complication, promoting the theory of "multiple hits" necessary for the development of secondary TMA [4], taking into account the possible immune-mediated mechanism of oxaliplatin-induced TMA, associated with a previous exposure to gemcitabine, which is known to induce TMA through a toxic mechanism secondary to endothelial damage [9].

Regardless of the pathophysiology, it is important to recognize early signs of this disease, due to its high mortality rate in case of not administering appropriate treatment. Despite the implication of a qualitative or quantitative deficiency of the enzyme ADAMTS13 in the pathophysiology of the disease, the clinicopathological findings of hemolytic anemia and thrombocytopenia in the context of certain diseases or exposure to medications or infections should lead to suspicion of this entity [5].

Once the diagnosis is established, it is necessary to start immediate therapy, which is generally based on plasma exchange, with which mortality can change from 90 to $20 \%[3,5]$. Additionally, the use of high-dose steroids is recommended concomitantly with plasma- 
pheresis, although it is important to note that some patients may improve with plasma exchange as single therapy [10].

Finally, TMA is a fatal entity, which occurs in patients on specific medications. Its causal association with the use of oxaliplatin is still enigmatic, with few reports in the literature on this association.

\section{Conclusion}

The clinical and paraclinical suspicion of TMA is crucial for the early initiation of therapies that can change the natural history of this not so rare complication. Despite the fact that we clearly know some diseases and medications that are associated with it, there are some circumstances such as in this case where its diagnosis should be considered, despite not having a clear association. There are case reports of oxaliplatin in which there is an evident association with the development of TMA, this being one of them. However, to our knowledge, this is the first case report in which there was previous exposure to a drug related to the development of TMA (gemcitabine), with subsequent administration of oxaliplatin, supporting the possible "multiple-hits" model.

\section{Statement of Ethics}

The manuscript has been sufficiently de-identified to protect the patient. However, informed consent was obtained for the publication of the case.

\section{Conflict of Interest Statement}

The authors have no conflicts of interest to declare.

\section{Funding Sources}

No funding was received.

None of the authors have disclosures relevant to this manuscript.

\section{Author Contributions}

All authors contributed to the editing of the manuscript.

M.C.F.-L. wrote the manuscript. E.C.B.-G. performed text review and corrections based on clinical experience.

\section{Karger'}


Fuentes-Lacouture/Barrera-Garavito: Thrombotic Microangiopathy during Treatment with Oxaliplatin

\section{References}

1 Tsai HM. Untying the knot of thrombotic thrombocytopenic purpura and atypical hemolytic uremic syndrome. Am J Med. 2013;126(3):200-9.

2 Scully M, Hunt BJ, Benjamin S, Liesner R, Rose P, Peyvandi F, et al. Guidelines on the diagnosis and management of thrombotic thrombocytopenic purpura and other thrombotic microangiopathies. Br J Haematol. 2012; 158(3):323-35.

3 Deford CC, Reese JA, Schwartz LH, Perdue JJ, Kremer Hovinga JA, Lämmle B, et al . Multiple major morbidities and increased mortality during long-term follow-up after recovery from thrombotic thrombocytopenic purpura. Blood. 2013;122(12):2023-142.

4 Bayer G, Von Tokarski F, Thoreau B, Bauvois A, Barbet C, Cloarec S, et al . Etiology and Outcomes of Thrombotic Microangiopathies. Clin J Am Soc Nephrol. 2019;14(4):557-66.

5 Arnold DM, Patriquin CJ, Nazy I. Thrombotic microangiopathies: a general approach to diagnosis and management. CMAJ. 2017;189(4):E153-9.

6 Gordon LI, Kwaan HC. Cancer- and drug-associated thrombotic thrombocytopenic purpura and hemolytic uremic syndrome. Semin Hematol. 1997;34(2):140-7.

7 Walter RB, Joerger M, Pestalozzi BC. Gemcitabine-associated hemolytic-uremic syndrome. Am J Kidney Dis. 2002;40(4):E16.

8 Baretta Z, Falci C, Piva E, Conte P. Fatal Oxaliplatin-Induced Thrombotic Thrombocytopenic Purpura: A Case Report. Clin Colorectal Cancer. 2013;12(4):294-6.

9 Hassane I, Corinne I, Vincent L, Lucille M, Isabelle T, Olivier R, et al. Gemcitabine-induced thrombotic microangiopathy: a systematic review, Nephrol Dial Transplant. 2006;21(11):3038-45.

10 George JN. How I treat patients with thrombotic thrombocytopenic purpura: 2010. Blood. 2010;116(20): $4060-9$. 